\title{
Study on the expression of p53 and MMP-2 in patients with lung cancer after interventional therapy
}

\author{
XUE-SONG ZHANG, KUI-YANG WANG, JIN-QI GAO, RUO-JIE LI, QING-BO GUAN and LEI SONG
}

Department of Intervention Therapy, The Second Hospital of Dalian Medical University, Dalian, Liaoning 116027, P.R. China

Received December 19, 2017; Accepted June 20, 2018

DOI: $10.3892 / \mathrm{ol} .2018 .9185$

\begin{abstract}
The aim of the study was to investigate the expression of tumor suppressor gene p53 and MMP-9 in non-small cell lung cancer (NSCLC) before and after chemotherapy, and investigate its association with the effect of chemotherapy and prognosis. Fifty-eight elderly NSCLC patients comprised the observation group. Reverse transcription-quantitative polymerase chain reaction (RT-qPCR) was used to detect the expression of p53 and MMP-9 in lung cancer tissues before and after chemotherapy. Immunohistochemistry and western blot analysis were used to detect the expression of p53 and MMP-9 proteins in NSCLC tissue before and after chemotherapy. Terminal deoxynucleotidyl transferase nick end-labeling (TUNEL) was used to detect apoptotic cells. The association between the effect of chemotherapy and the expression of p53 and MMP-9 in lung cancer tissues was analysed. RT-qPCR results showed that the expression of p53 and MMP-2 mRNA in the tumor tissue after chemotherapy was significantly lower than that in the tumor tissue before chemotherapy. Western blot analysis revealed that the expression of p53 and MMP-2 protein in the tumor tissue after chemotherapy was significantly decreased. The positive expression of p53 and MMP-2 in lung cancer tissues before chemotherapy was 76.25 and $71.25 \%$, respectively, and were reduced to 27.50 and $23.75 \%$, respectively, after chemotherapy. After chemotherapy, the positive rates of p53 and MMP-2 were significantly lower than those before chemotherapy. TUNEL results showed that the apoptosis index increased significantly after chemotherapy. Efficiency of chemotherapy in patients with a negative expression of p53 and MMP-2 in lung cancer before chemotherapy was significantly higher than that in patients with a positive p53 and MMP-2 expression. A significant difference was found in the expression levels of p53 and MMP-2 in lung cancer before and after chemotherapy. The
\end{abstract}

Correspondence to: Dr Xue-Song Zhang, Department of Intervention Therapy, The Second Hospital of Dalian Medical University, 467 Zhongshan Road, Shahekou, Dalian, Liaoning 116027, P.R. China

E-mail: bz088gc@163.com

Key words: p53, MMP-2, non-small cell lung cancer, chemotherapy findings of the present study indicate that the expression levels of p53 and MMP-2 can be used as a predictor of chemotherapy sensitivity.

\section{Introduction}

As one of the most common malignant tumors, lung cancer is characterized by high morbidity and mortality rates (1). There are many types of lung cancer, and the number of patients with non-small cell lung cancer (NSCLC) is the largest, accounting for approximately $80 \%$ of the total number of patients with lung cancer (2). Due to the complex diagnosis of lung cancer and imperceptible early symptoms, $70-80 \%$ of patients with lung cancer are initially diagnosed at an advanced stage (3).

The p53 gene was originally considered a tumor suppressor gene that exerts an antitumor effect by regulating cell growth and apoptosis. After mutation, the wild-type p53 gene becomes the mutant-type p53 gene with an apoptosis-inhibiting effect, which can stimulate and promote the growth of tumor cells, losing the normal antitumor effect (4). Previous findings have confirmed that p53 gene mutates in $50 \%$ of human tumor tissues, and can be used as a tumor biomarker (5). Matrix metalloproteinase (MMP) is a zinc ion-dependent protease family, of which MMP-2 is a major member (6,7). MMP-2 is mainly secreted by tumor cells and interstitial cells in the form of zymogen. After hydrolysis and activation, MMP-2 can degrade type IV collagen in the basement membrane. When the collagen in the basement membrane is degraded and destroyed, MMP-2 further affects the hindering effect of the basement membrane on tumor cells (8). MMP-2 has been shown to play a key role in degrading the extracellular matrix and promoting tumor invasion and metastasis $(9,10)$.

In recent years, surgery after adjuvant chemotherapy has become a new strategy for the treatment of lung cancer, but there are no unified criteria for the chemotherapeutic effect clinically at present. Therefore, in this study, the effects of interventional chemotherapy on the protein expressions of $\mathrm{p} 53$ and MMP-2 in tumor tissues of NSCLC patients, and their correlations with tumor cell apoptosis in patients and chemotherapeutic effect were investigated. The mRNA expression levels of p53 and MMP-2 in tumor tissues before and after chemotherapy were detected via reverse transcription-quantitative polymerase chain reaction (RT-qPCR). Changes in the protein expressions of p53 and MMP-2 in tumor tissues 
and tissue cell apoptosis before and after chemotherapy were further studied, and the correlations of the expression of p53 and MMP-2 in tumor tissues of patients with chemotherapeutic effect before chemotherapy were analyzed combined with clinical data.

\section{Materials and methods}

Materials. Ribonucleic acid (RNA) extraction kit (Invitrogen, Carlsbad, CA, USA); primer synthesis, RT kit, real-time fluorescence quantitative PCR kit (Takara Biotechnology Co., Ltd., Dalian, China); p53, MMP-2, glyceraldehyde-3-phosphate dehydrogenase (GAPDH) primary antibodies and horseradish peroxidase (HRP)-labeled secondary antibodies (Proteintech, Wuhan, China); terminal deoxynucleoitidyl transferase-mediated dUTP nick end-labeling (TUNEL) apoptosis assay kit, DAPI staining solution and Triton X-100 (Nanjing KeyGen Biotech, Nanjing, China); protein extraction kit, and bicinchoninic acid (BCA) protein assay kit (Beyotime Biotechnology, Nantong, China) were used in the present study.

A total of 80 elderly patients with NSCLC aged $\geq 65$ years, admitted to the Department of Surgery, The Second Hospital of Dalian Medical University (Dalian, China) were selected, including 44 males and 36 females with an average age of 75.5 years. Tissue specimens were obtained from all the patients via bronchoscopy and computed tomography (CT)-guided biopsy, and the patients were diagnosed with NSCLC via pathological examination but received no chemotherapy. Patients were treated with navelbine + cisplatin chemotherapy. At 2 weeks after chemotherapy, blood and urine routine, hepatic and renal functions, cardiac function and chest CT were reviewed. Patients underwent surgery at 2 weeks after chemotherapy, if there were no surgical contraindications. Specimens after chemotherapy were used as postoperative pathological specimens; one part of the specimens before and after chemotherapy was fixed with formalin and embedded into paraffin, while the other part was directly cryopreserved at $-80^{\circ} \mathrm{C}$. In the study, the acquisition of all the specimens was approved by the Clinical Ethics Committee of The Second Hospital of Dalian Medical University (Dalian, China). All the patients enrolled or their families signed the informed consent.

Detection of $m R N A$ expression of $p 53$ and MMP-2 in tumor tissue specimens in patients before and after chemotherapy via $R T$-qPCR. Approximately $100 \mathrm{mg}$ tumor tissue specimens before and after chemotherapy were taken from each patient, and the total RNA was extracted from the tissues to be tested according to the protocol of the RNA extraction kit. Specimens with an absorbance (A) 260/A280 ratio of 1.8-2.0 were selected for subsequent experiments. Reverse transcription was performed according to the protocol of the RT kit to obtain the complementary DNA (cDNA). With cDNA as a template, the mRNA expression of p53 and MMP-2 was detected via quantitative PCR, and GAPDH was used as an internal control. Primer sequences are shown in Table I. Reaction conditions were as follows: $95^{\circ} \mathrm{C}$ for $10 \mathrm{~min}, 95^{\circ} \mathrm{C}$ for $15 \mathrm{sec}, 60^{\circ} \mathrm{C}$ for $1 \mathrm{~min}$, and amplification for 35 cycles. The cycle threshold $(\mathrm{Cq})$ value was output from the instrument software, and the relative expression level was calculated
Table I. RT-qPCR primer sequences

\begin{tabular}{lll}
$\begin{array}{l}\text { Gene } \\
\text { name }\end{array}$ & $\begin{array}{c}\text { Primer } \\
\text { name }\end{array}$ & Primer sequence \\
\hline$p 53$ & F: & 5'-TGCGTGTGGAGTATTTGGATG-3' \\
& R: & 5'-TGGTACAGTCAGAGCCAACCTC-3' \\
MMP-2 & F: & 5'-CTCATCGCAGATGCCTGGAA-3' \\
& R: & 5'-TTCAGGTAATAGGCACCCTTGAAGA-3' \\
GAPDH & F: & 5'-GCACCGTCAAGGCTGAGAAC-3' \\
& R: & 5'-TGGTGAAGACGCCAGTGGA-3' \\
& & \\
\hline
\end{tabular}

F, forward; R, reverse.

according to the formula: $\Delta \mathrm{Cq}$ (target gene) $=\mathrm{Cq}$ (target gene) - $\mathrm{Cq}$ (control gene)

Detection of protein expression of p53 and MMP-2 in tumor tissue specimens in patients before and after chemotherapy via western blot analysis. An appropriate number of frozen tissue specimens of patients were taken, lysed using a tissue lysis buffer, and centrifuged at $3000 \mathrm{x} \mathrm{g}$ at $4^{\circ} \mathrm{C}$ for $15 \mathrm{~min}$, prior to the supernatant being collected. The concentration of protein extracted was determined using a BCA kit, and $50 \mu \mathrm{g}$ protein was subjected to sodium dodecyl sulfate polyacrylamide gel electrophoresis (SDS-PAGE), transferred onto a polyvinylidene fluoride (PVDF) membrane using the wet method, sealed using the blocking solution at room temperature for $2 \mathrm{~h}$, and added with rabbit anti-human p53 and MMP-2 primary monoclonal antibodies (1:1,000; cat. nos. 2527 and 87809, respectively; both obtained from Cell Signaling Technology, Inc., Danvers, MA, USA) for incubation at $4^{\circ} \mathrm{C}$ overnight. The membrane was washed with TTBS 3 times and added with anti-rabbit IgG, HRP-linked secondary polyclonal antibody (1:2,000; cat. no. 7074; Cell Signaling Technology, Inc.) for incubation at room temperature for $2 \mathrm{~h}$. Color was developed using the electrochemiluminescence (ECL) solution in the dark, followed by scanning and recording with a gel imager. The gray scale was analyzed and compared with GADPH as an internal reference.

Detection of protein expression of p53 and MMP-2 in tumor tissue specimens in patients before and after chemotherapy via IHC. Tissue sections were routinely dewaxed, followed by antigen retrieval using the citrate buffer solution via microwave. Normal serum blocking solution was dropwise added to block the sections, p53 and MMP-2 primary antibodies (diluted at 1:100) were added, and the sections were placed in a refrigerator at $4^{\circ} \mathrm{C}$ overnight. The sections were washed with phosphate-buffered saline (PBS) 3 times, added with the biotin-labeled secondary antibodies for incubation for another 15 min, and washed again with PBS 3 times, followed by color development in the dark with DAB solution, re-staining with hematoxylin, sealing via gum, observation and photography under a microscope (TE2000-U, Nikon Corp., Tokyo, Japan).

Staining results were evaluated in randomly selected areas: the expression intensity was graded and scored according to the staining depth and percentage of positive 

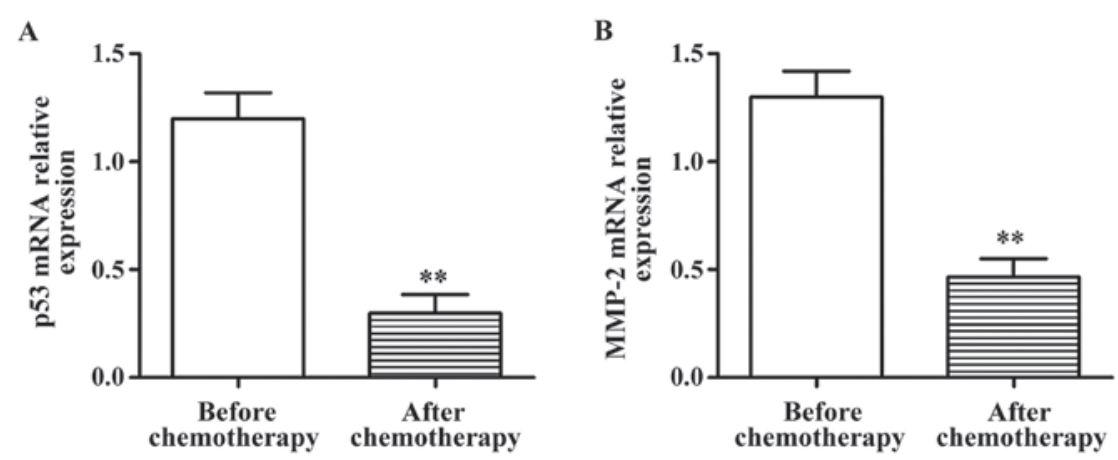

Figure 1. Detection of mRNA expression of p53 and MMP-2 in tissue specimens of elderly patients with lung cancer before and after chemotherapy via RT-PCR. Compared with those before chemotherapy, the mRNA expression of (A) p53 and (B) MMP-2 in tissues of elderly patients with lung cancer after chemotherapy are significantly decreased; * $\mathrm{p}<0.01$.

cells. Staining intensity was: no staining (0 point), pale yellow (1 point), brown yellow (2 points) and dark brown (3 points); the number of positive cells in high-power fields $(\mathrm{x} 400)$ was: $<5 \%$ (0 point), $5-25 \%$ ( 1 point), $26-50 \%$ ( 2 points) and $>50 \%$ ( 3 points). If the two points were added up, a score of $>3$ points indicated a positive expression, while that of $\leq 2$ points indicated a negative expression. Results were statistically analyzed.

Detection of changes in apoptosis of tumor cells before and after chemotherapy via TUNEL assay. Sections were completely immersed in $4 \%$ paraformaldehyde fixing solution for fixation for $30 \mathrm{~min}$, permeabilized with $1 \%$ Triton X-100 and rinsed 3 times. DNase I reaction solution (100 $\mu \mathrm{l})$ already prepared was dropwise added onto the sections selected for incubation at $37^{\circ} \mathrm{C}$ for $30 \mathrm{~min}$, and the sections were rinsed with PBS 3 times. The sections were then added with $50 \mu \mathrm{l}$ TdT reaction solution, placed into a warm box, incubated in the dark at $37^{\circ} \mathrm{C}$ for $60 \mathrm{~min}$, and rinsed again with PBS 3 times in the dark. The sections were added with streptavidin-tetraethyl rhodamine isothiocyanate (TRITC) labeling solution, placed into a wet box, incubated in the dark at $37^{\circ} \mathrm{C}$ for $30 \mathrm{~min}$, and rinsed again with PBS 3 times in the dark. After re-staining via DAPI staining solution, the sections were incubated in the dark for $10 \mathrm{~min}$, and rinsed with PBS 3 times in the dark, followed by observation and photography under a fluorescence microscope (Olympus Corporation, Tokyo, Japan).

Correlation of p53 and MMP-2 protein expressions with chemotherapeutic effect. At 2 weeks after surgery, the chemotherapeutic effect was evaluated according to the therapeutic evaluation criteria of solid tumor of WHO. The effects were divided into four types: complete remission, partial remission, stable disease and progressive disease. Complete remission and partial remission indicated the effectiveness, while stable disease and progressive disease indicated the ineffectiveness, followed by data statistics and analysis.

Statistical analysis. Statistical Product and Service Solutions (SPSS) 17.0 software (International Business Machines Corporation, Armonk, NY, USA) was used for data processing in the present study. Measurement data were presented as mean \pm standard deviation, and Student's t-test was used for the intergroup comparison; Chi-square analysis was used for the comparison of enumeration data between two groups. $\mathrm{P} \leq 0.05$ suggested that the difference was statistically significant.

\section{Results}

mRNA expression of p53 and MMP-2 in tissue specimens of patients before and after chemotherapy. Frozen tumor tissues and paracarcinoma normal tissues were taken from patients before and after chemotherapy to detect the mRNA expression of p53 and MMP-2 via RT-PCR. The results showed that the mRNA expression of p53 and MMP-2 in lung cancer tissues after chemotherapy were significantly lower than those before chemotherapy, and the differences were statistically significant $(\mathrm{p}<0.01)$ (Fig. 1).

Protein expression of 553 and MMP-2 in tissue specimens of patients before and after chemotherapy. The protein expression of p53 and MMP-2 in tissue specimens of patients before and after chemotherapy were further detected via western blot analysis. The results showed that the protein expression of p53 and MMP-2 in tumor tissues of elderly patients with lung cancer after chemotherapy was significantly decreased compared with those before chemotherapy $(\mathrm{p}<0.01)$ (Fig. 2).

Detection of the protein expression of p53 and MMP-2 in tissue specimens of patients before and after chemotherapy via IHC. IHC results revealed that $\mathrm{p} 53$ protein was located in the nucleus, while MMP-2 protein was located in the cytoplasm, showing the diffuse or scattered distribution (Fig. 3). Statistical analysis showed that the positive expression rates of p53 and MMP-2 in lung cancer tissues of 80 patients were $76.25 \%(61 / 80)$ and $71.25 \%$ (57/80) before chemotherapy, respectively, and $27.50 \%(22 / 80)$ and $23.75 \%$ (19/80) after chemotherapy, respectively. The positive expression rates of p53 and MMP-2 after chemotherapy were significantly lower than those before chemotherapy, and the differences were statistically significant $(\mathrm{p}<0.01)$.

Changes in cell apoptosis in patients before and after chemotherapy. Results of the TUNEL assay showed that the green fluorescence-stained nucleus indicated the positive cells, namely apoptotic cells. Compared with that before chemotherapy, the number of apoptotic cells in tumor tissues of 
$\mathbf{A}$
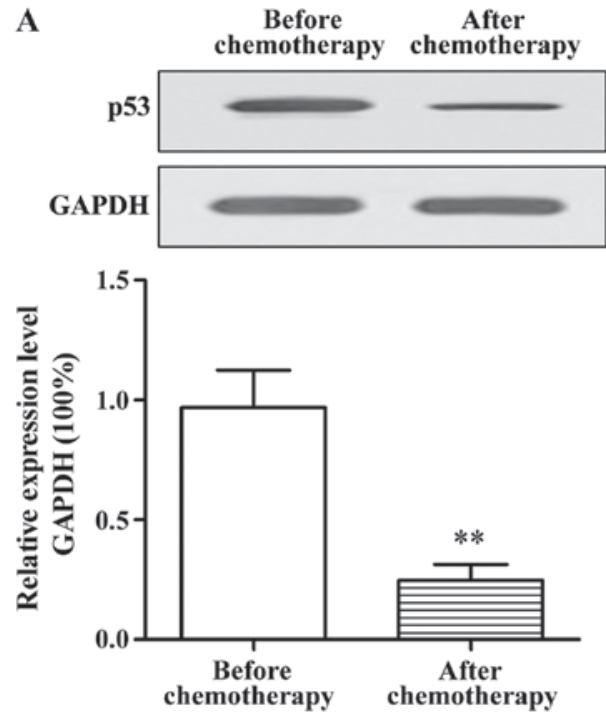

B
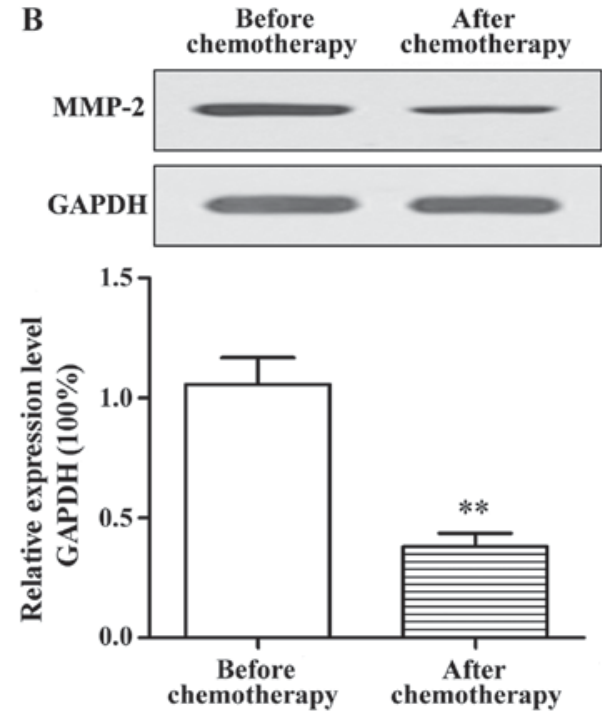

Figure 2. Detection of the protein expression of p53 and MMP-2 in tissue specimens of elderly patients with lung cancer before and after chemotherapy via western blot analysis. Compared with those before chemotherapy, the protein expression of (A) p53 and (B) MMP-2 in tissues of elderly patients with lung cancer after chemotherapy are significantly decreased; ${ }^{*} \mathrm{p}<0.01$.
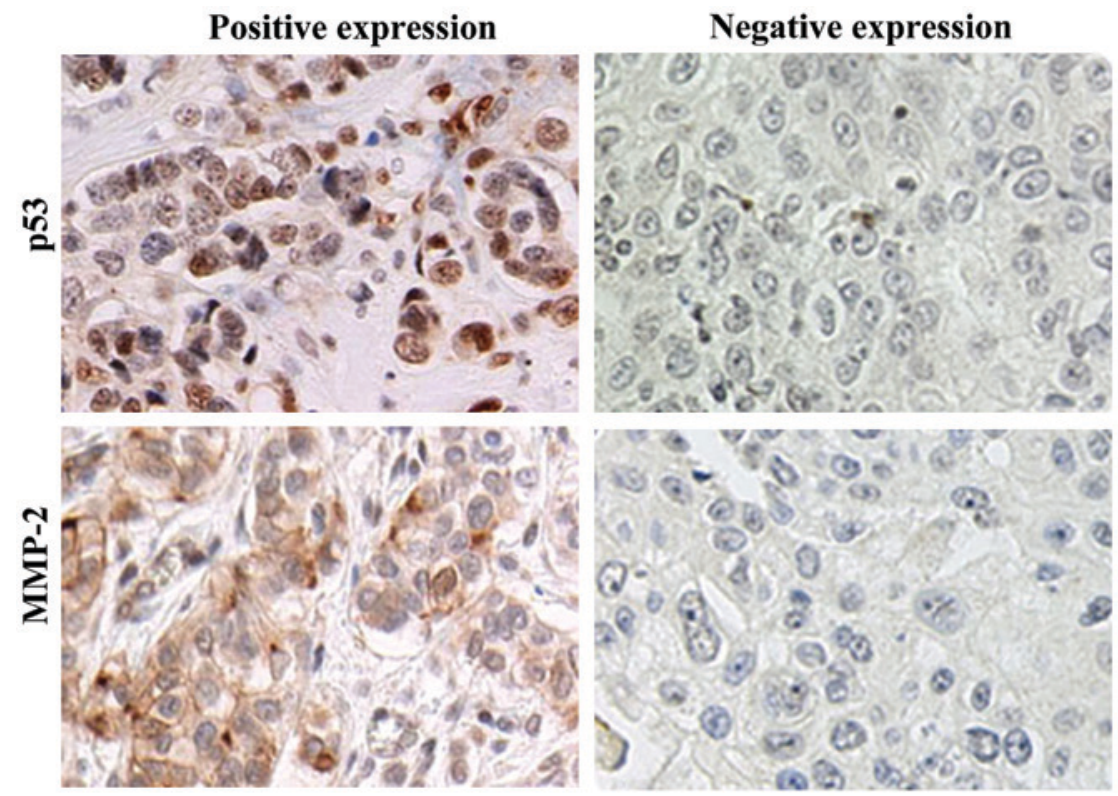

Figure 3. Detection of protein expression of p53 and MMP-2 in tissue specimens of elderly patients with lung cancer before and after chemotherapy via IHC. The 53 protein is located in the nucleus, while MMP-2 protein is located in the cytoplasm, showing the diffuse or scattered distribution.
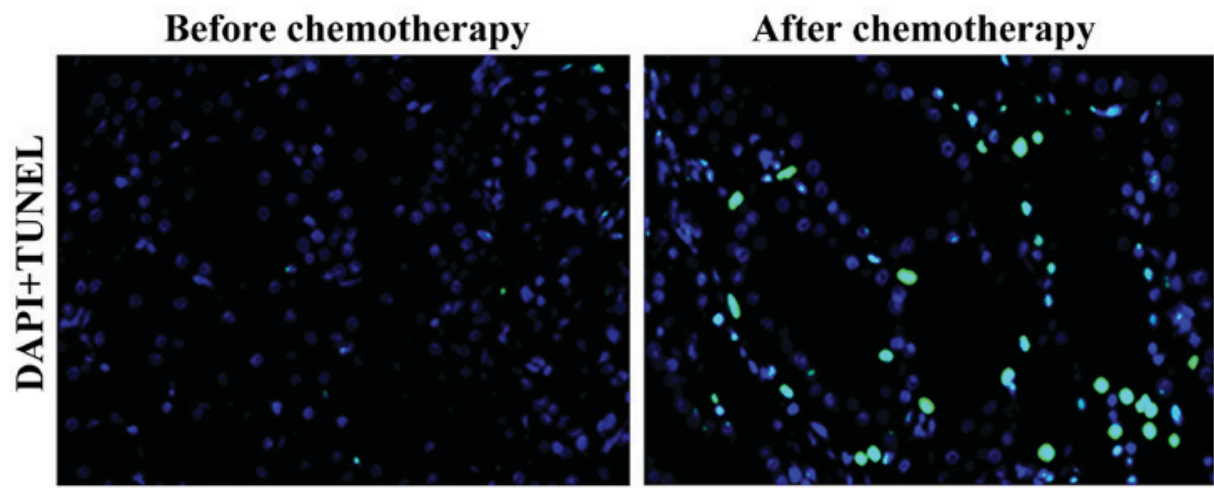

Figure 4. Detection of cell apoptosis in elderly patients with lung cancer before and after chemotherapy via TUNEL staining. Compared with that before chemotherapy, the number of apoptotic cells in tumor tissues of elderly patients with lung cancer is significantly increased after chemotherapy. 
Table II. Correlations of p53 and MMP-2 expressions in lung cancer tissues before chemotherapy with chemotherapeutic effect $[\mathrm{n}(\%)]$.

\begin{tabular}{|c|c|c|c|c|}
\hline \multirow[b]{2}{*}{ Chemotherapeutic effect } & \multicolumn{2}{|c|}{ p53 } & \multicolumn{2}{|c|}{ MMP-2 } \\
\hline & Positive & Negative & Positive & Negative \\
\hline Effective & $32(52.46)$ & $15(78.95)$ & $29(50.88)$ & $18(78.26)$ \\
\hline Ineffective & 29 & 4 & 28 & 5 \\
\hline$\chi^{2}$ & \multicolumn{2}{|c|}{4.19} & \multicolumn{2}{|c|}{5.07} \\
\hline p-value & \multicolumn{2}{|c|}{$<0.05$} & \multicolumn{2}{|c|}{$<0.05$} \\
\hline
\end{tabular}

elderly patients with lung cancer was significantly increased after chemotherapy (Fig. 4).

Correlations of p53 and MMP-2 expressions in lung cancer tissues before chemotherapy with chemotherapeutic effect. The effective rates of chemotherapy in patients with a negative expression of p53 and MMP-2 in lung cancer tissues before chemotherapy were 78.95 and $78.26 \%$, respectively, while those in patients with positive expressions of p53 and MMP-2 in lung cancer tissues before chemotherapy were 52.46 and $50.88 \%$, respectively. The effective rates of chemotherapy in patients with negative expressions of p53 and MMP-2 in lung cancer tissues before chemotherapy were significantly higher than those in patients with positive expressions, and the differences were statistically significant $(\mathrm{p}<0.01)$ (Table II).

\section{Discussion}

Lung cancer has a high morbidity rate, and its mortality rate ranks first in malignant tumors in China (11). There are no obvious clinical symptoms of lung cancer in the early stage, thus patients have been in the advanced stage when diagnosed. Approximately $40 \%$ of patients with newly diagnosed lung cancer are elderly, aged above 65 years (12).

The p53 gene is located on human chromosome 17p13, which is a kind of tumor suppressor gene and pro-apoptosis gene. Wild-type p53 plays a key role in cellular gene transcription, cell cycle regulation, apoptosis, cell proliferation and differentiation (13-15). Previous findings showed that the mutation of p53 gene is closely related to the occurrence and development of liver cancer $(15,16)$. The content of wild-type p53 protein is very low in normal cells, and it has a short half-life period, and cannot be detected via IHC and other commonly used methods. However, the mutant-type p53 protein has a longer half-life period and a higher expression level, and is easily detected $(17,18)$.

The MMP-2 gene is located on human chromosome $16 q 21$, which can degrade, not only type IV collagen in basement membrane, but also type V, VI and X collagen and gelatin following activation. Extracellular matrix and basement membrane act as natural barriers in tumor invasion and diffusion processes; thus, passing through this layer of tissue barrier is a key step in the tumor cell metastasis (19). Additionally, tumor cells can specifically express MMP-2 highly to degrade type IV collagen, and destroy the tissue barrier, thereby promoting tumor cell invasion and metastasis (20).

In this study, the effects of interventional chemotherapy on the protein expression of p53 and MMP-2 in tumor tissues of NSCLC patients, and their correlations with tumor cell apoptosis in patients and chemotherapeutic effect were investigated. The mRNA expression levels of p53 and MMP- 2 in tumor tissues before and after chemotherapy were detected via RT-qPCR and the results showed that the mRNA expression levels of p53 and MMP-2 in lung cancer tissues after chemotherapy were significantly lower than those prior to chemotherapy. The protein expression levels of p53 and MMP-2 in tumor tissues before and after chemotherapy were further studied. The results showed that the protein expression of p53 and MMP-2 in tumor tissues of elderly patients with lung cancer after chemotherapy were significantly decreased, and the positive expression rates of p53 and MMP-2 after chemotherapy were significantly lower than those before chemotherapy. In addition, the effect of chemotherapy on apoptosis of tissue cells was studied via TUNEL assay. The results revealed that the number of apoptotic cells in tumor tissues of elderly patients with lung cancer was significantly higher after chemotherapy than those before chemotherapy. It was also found combined with clinical data analysis that the effective rates of chemotherapy in patients with negative expressions of p53 and MMP-2 in lung cancer tissues before chemotherapy were significantly higher than those in patients with positive expressions.

In conclusion, the results of the present study preliminarily demonstrate that the expression levels of p53 and MMP-2 can be used to predict the sensitivity of elderly patients with lung cancer to chemotherapy drugs, and both p53 and MMP-2 may serve as molecular markers for predicting the sensitivity of lung cancer to chemotherapy, better serving the clinical treatment.

\section{Acknowledgements}

Not applicable.

\section{Funding}

No funding was received.

\section{Availability of data and materials}

The datasets used and/or analyzed during the current study are available from the corresponding author on reasonable request. 


\section{Authors' contributions}

XSZ and KYW performed PCR. JQG and RJL were responsible for western blot analysis. QBG and LS helped with IHC. All authors read and approved the final manuscript.

\section{Ethics approval and consent to participate}

In the study, the acquisition of all the specimens was approved by the Clinical Ethics Committee of The Second Hospital of Dalian Medical University (Dalian, China). All the patients enrolled or their families signed the informed consent.

\section{Patient consent for publication}

Not applicable.

\section{Competing interests}

The authors declare that they have no competing interests.

\section{References}

1. Chen QY, Zheng Y, Jiao DM, Chen FY, Hu HZ, Wu YQ, Song J, Yan J, Wu LJ and Lv GY: Curcumin inhibits lung cancer cell migration and invasion through Racl-dependent signaling pathway. J Nutr Biochem 25: 177-185, 2014

2. Feng B, Zhang K, Wang R and Chen L: Non-small-cell lung cancer and miRNAs: Novel biomarkers and promising tools for treatment. Clin Sci (Lond) 128: 619-634, 2015

3. Johnson DH: Locally advanced, unresectable non-small cell lung cancer: New treatment strategies. Chest 117 (Suppl 1): S123-S126, 2000.

4. Deveraux QL and Reed JC: IAP family proteins - suppressors of apoptosis. Genes Dev 13: 239-252, 1999.

5. Holcik M, Gibson H and Korneluk RG: XIAP: Apoptotic brake and promising therapeutic target. Apoptosis 6: 253-261, 2001.

6. Chia CY, Kumari U and Casey PJ: Breast cancer cell invasion mediated by $\mathrm{G} \alpha 12$ signaling involves expression of interleukins- 6 and -8, and matrix metalloproteinase-2. J Mol Signal 9: 6, 2014.

7. Stoeltzing O, Ahmad SA, Liu W, McCarty MF, Wey JS, Parikh AA, Fan F, Reinmuth N, Kawaguchi M, Bucana CD, et al: Angiopoietin-1 inhibits vascular permeability, angiogenesis, and growth of hepatic colon cancer tumors. Cancer Res 63: 3370-3377, 2003.

8. Zhang W, Wang F, Xu P, Miao C, Zeng X, Cui X, Lu C, Xie H, Yin $\mathrm{H}$, Chen $\mathrm{F}$, et al: Perfluorooctanoic acid stimulates breast cancer cells invasion and up-regulates matrix metalloproteinase-2/-9 expression mediated by activating NF- $\kappa$ B. Toxicol Lett 229: 118-125, 2014.
9. Iochmann S, Bléchet C, Chabot V, Saulnier A, Amini A, Gaud G, Gruel Y and Reverdiau P: Transient RNA silencing of tissue factor pathway inhibitor-2 modulates lung cancer cell invasion. Clin Exp Metastasis 26: 457-467, 2009.

10. Safranek J, Pesta M, Holubec L, Kulda V, Dreslerova J, Vrzalova J, Topolcan O, Pesek M, Finek J and Treska V: Expression of MMP-7, MMP-9, TIMP-1 and TIMP-2 mRNA in lung tissue of patients with non-small cell lung cancer (NSCLC) and benign pulmonary disease. Anticancer Res 29: 2513-2517, 2009.

11. Shi Y and Sun Y: Medical management of lung cancer: Experience in China. Thorac Cancer 6: 10-16, 2015.

12. Gridelli C, Aapro M, Ardizzoni A, Balducci L, De Marinis F, Kelly K, Le Chevalier T, Manegold C, Perrone F, Rosell R, et al: Treatment of advanced non-small-cell lung cancer in the elderly: Results of an international expert panel. J Clin Oncol 23: 3125-3137, 2005

13. Hu W, Wang F, Tang J, Liu X, Yuan Z, Nie C and Wei Y: Proapoptotic protein Smac mediates apoptosis in cisplatin-resistant ovarian cancer cells when treated with the anti-tumor agent AT101. J Biol Chem 287: 68-80, 2012.

14. Hussain AR, Uddin S, Ahmed M, Bu R, Ahmed SO, Abubaker J, Sultana M, Ajarim D, Al-Dayel F, Bavi PP, et al: Prognostic significance of XIAP expression in DLBCL and effect of its inhibition on AKT signalling. J Pathol 222: 180-190, 2010.

15. Shangary S and Wang S: Targeting the MDM2-p53 interaction for cancer therapy. Clin Cancer Res 14: 5318-5324, 2008.

16. Shangary S, Qin D, McEachern D, Liu M, Miller RS, Qiu S, Nikolovska-Coleska Z, Ding K, Wang G, Chen J, et al: Temporal activation of $\mathrm{p} 53$ by a specific MDM2 inhibitor is selectively toxic to tumors and leads to complete tumor growth inhibition. Proc Natl Acad Sci USA 105: 3933-3938, 2008.

17. Scoumanne A and Chen X: Protein methylation: A new mechanism of p53 tumor suppressor regulation. Histol Histopathol 23: 1143-1149, 2008.

18. Lu C and El-Deiry WS: Targeting p53 for enhanced radio- and chemo-sensitivity. Apoptosis 14: 597-606, 2009.

19. Fernandez-Garcia B, Eiró N, Marín L, González-Reyes S, González LO, Lamelas ML and Vizoso FJ: Expression and prognostic significance of fibronectin and matrix metalloproteases in breast cancer metastasis. Histopathology 64: 512-522, 2014.

20. Parsons SL, Watson SA, Collins HM, Griffin NR, Clarke PA and Steele RJ: Gelatinase (MMP-2 and -9) expression in gastrointestinal malignancy. Br J Cancer 78: 1495-1502, 1998.

This work is licensed under a Creative Commons Attribution-NonCommercial-NoDerivatives $\quad 4.0$ International (CC BY-NC-ND 4.0) License. 